\title{
Growth Cone Collapse Induced by Semaphorin 3A Requires 12/15-Lipoxygenase
}

\author{
Keith Mikule, Jesse C. Gatlin, Becky A. de la Houssaye, and Karl H. Pfenninger \\ Department of Cellular and Structural Biology, University of Colorado School of Medicine, Denver, Colorado 80262
}

Detection of a repellent factor, such as a semaphorin (Sema), causes localized collapse of the growth cone and directs the neurite away from the repellent. Growth cone collapse results from concomitant cytoskeletal rearrangements and detachment of adhesion sites from the extracellular matrix, via mostly unknown signaling mechanisms. In cultures of dorsal root ganglion neurons, we found that Sema3A treatment stimulates the synthesis of the eicosanoid, 12(S)-hydroxyeicosatetraenoic acid (HETE), whereas Sema3A-induced growth cone collapse is prevented when 12(S)-HETE synthesis is blocked with an inhibitor of 12/15-lipoxygenase (LO). Exogenously applied product of 12/15-LO, 12(S)-HETE, mimics Sema3A-induced collapse. As observed by interference reflection and confocal microscopy, 12(S)-HETE causes the loss of growth cone adhesion sites. The adhesion site effect seems partially independent of the actin cytoskeleton because growth cones treated with Sema3A and 12/15-LO inhibitor remain spread despite actin cytoskeleton loss. These studies demonstrate that 12/15-LO activity is a necessary step in Sema3A collapse signaling in growth cones and suggest a mechanism for its action.

Key words: neurite pathfinding; lipoxygenase; semaphorin; growth cone adhesion; growth cone collapse; eicosanoid signaling
Accurate axonal targeting requires that growth cones navigate precisely along pathways defined by attractive and repulsive cues (Tessier-Lavigne and Goodman, 1996). Guidance cues are detected by receptor-rich growth cone filopodia that continuously survey their microenvironment (Lauffenburger and Horwitz, 1996). Filopodial contact with positive chemotactic factors stimulates growth cone translocation, which results from a cycle involving F-actin assembly driving lamellipodial extension, distal adhesion of the newly formed lamellipodia (to form an adhesion site), and proximal de-adhesion of the growth cone from the substrate (Richards et al., 1997). In contrast, filopodial contact with a repulsive factor, such as thrombin or a semaphorin (Sema), triggers de-adhesion and growth cone collapse, followed by retraction (Keynes and Cook, 1992; de la Houssaye et al., 1999; Fritsche et al., 1999; Fournier et al., 2000a).

Semas affect axonal guidance, cell migration, and neoplastic transformation (Tessier-Lavigne and Goodman, 1996; Soker et al., 1998; Behar et al., 1999). Sema3A is a class III secreted polypeptide that binds and activates a receptor complex consisting of neuropilin-1 (NP-1) and plexin A (Takahashi et al., 1999). When applied as a gradient, Sema3A causes growth cones to turn away from the source; incubation of cultured neurites with Sema3A triggers collapse of their growth cones (Fan and Raper, 1995). Sema3 $A^{-1-}$ mice exhibit excessive growth of peripheral dorsal root ganglion (DRG) axons, reflecting the lack of normal Sema3A repulsion in vivo (Taniguchi et al., 1997). Several molecules involved in growth cone collapse have been identified. They include RhoA, Rac1, Cdc42, Rnd, and "collapse response medi-

Received Aug. 3, 2001; revised March 15, 2002; accepted March 22, 2002.

This work was supported by National Institutes of Health Grants NS24672 and NS41029 to K.H.P. We thank Dr. W. J. Betz for access to his confocal microscope and Steve Fadul for his help with this technology.

Correspondence should be addressed to Dr. Karl H. Pfenninger, Department of Cellular and Structural Biology, University of Colorado School of Medicine, B111 4200 East 9th Avenue, Denver, CO 80262. E-mail: Karl.Pfenninger@uchsc.edu. Copyright (C) 2002 Society for Neuroscience $0270-6474 / 02 / 224932-10 \$ 15.00 / 0$ ator proteins" (CRMPs) and seem to be associated with the cytoskeletal effects induced by Sema3A (Goshima et al., 1995; Jin and Strittmatter, 1997; Zanata et al., 2002). Additionally, intracellular levels of cGMP have been shown to influence Sema3Ainduced growth cone turning (Song et al., 1998). However, the signaling pathway leading to Sema3A-induced collapse is mostly unknown.

Amoeboid systems like growth cones, polymorphonuclear leukocytes, and platelets possess high levels of free arachidonic acid (AA) (Mazurov et al., 1983; Doukas et al., 1988; Negre-Aminou and Pfenninger, 1993). The free AA found in growth cones results from the action of at least two different cytoplasmic forms of phospholipase $\mathrm{A}_{2}\left(\mathrm{CPLA}_{2}\right)$ (Negre-Aminou and Pfenninger, 1993). We also demonstrated that isolated growth cones treated with the repellent thrombin greatly increase AA levels by activation of $\mathrm{cPLA}_{2}$. Although most $\mathrm{AA}$ is rapidly reincorporated into phospholipid, some of it is converted by leukocyte 12/15lipoxygenase (LO) to 12(S)- and 15(S)-hydroperoxyeicosatetraenoic acid, which is subsequently reduced to $12(\mathrm{~S})$ - and 15(S)-hydroxyeicosatetraenoic acid (HETE) (Dailey and Imming, 1999; Yamamoto et al., 1999). Experiments on growth cones in culture show that $12 / 15$-LO is necessary for thrombin-induced collapse, and 12(S)-HETE was shown to be sufficient for growth cone collapse in cortical neurons (de la Houssaye et al., 1999). The present studies on DRG neurons test the hypothesis that at least one of the Semas, Sema3A, also uses a signaling pathway involving 12/15-LO to trigger growth cone collapse and that this pathway affects growth cone adhesion.

\section{MATERIALS AND METHODS}

Materials. Experiments were performed with recombinant Sema3A used either as partially purified protein or as Sema3A-enriched culture supernatant of stably expressing 293 cells. Enrichment was achieved by concentrating the culture medium on a Centriplus membrane (Millipore Corporation, Bedford, MA; 50,000 MW cutoff). Partially purified Sema3A and Sema3A-expressing 293 cells were the generous gift of Dr. 
Yuling Luo (Exelixis Pharmaceuticals, Inc., South San Francisco, CA). Sema3A preparations were calibrated based on the degree of the collapse response and compared with that achieved with the partially purified factor ( $\sim 1 \mathrm{nM})$. Antibody to NP-1 was a gift from Dr. Alex Kolodkin (The Johns Hopkins University School of Medicine). Cinnamyl-3,4dihydroxy- $\alpha$-cyanocinnamate (CDC), 12(S)-HETE, and indomethacin were purchased from Biomol. 5(R)-HETE was obtained from Calbiochem (LaJolla, CA). NGF was from Collaborative Biomedical Products. All other chemicals, unless stated otherwise, were from Sigma (St. Louis, MO) and of the highest quality obtainable.

Neuron culture. DRGs were dissected from embryonic day 15 Sprague Dawley rats and cultured on laminin-coated cover glass (Assistant Brand; Carolina Biological Supply Co., Burlington, NC) in B27 neurobasal medium (NB) supplemented with $10 \%$ FBS and $100 \mathrm{ng} / \mathrm{ml} \mathrm{NGF}$. The cultures were incubated at $37^{\circ} \mathrm{C}$ in $5 \% \mathrm{CO}_{2}$ in air. Sprouting neurons were observed readily on day 1 . Long neurites were present on day 2 or 3 and used for experiments.

12(S)-HETE immunobinding assay. DRG neurons were cultured as described above and used for experimentation on day 1.5. Cultures that received $\mathrm{CDC}$ did so $30 \mathrm{~min}$ before challenge with Sema3A. For $3 \mathrm{~min}$ before extraction, cultures were incubated at $37^{\circ} \mathrm{C}$ with Sema3A, CDC $(10 \mu \mathrm{M}), \mathrm{CDC}(10 \mu \mathrm{M})$ followed by Sema3A, or left untreated. During extraction with detergent-containing buffer, 12(S)-HETE was assayed quantitatively by immunobinding kit (Assay Designs, Ann Arbor, MI) according to the instructions of the supplier. The assay uses a polyclonal antibody to 12(S)-HETE to bind, in a competitive manner, the 12(S)HETE contained within a sample or an alkaline phosphatase-12(S)HETE conjugate. This assay is capable of measuring $\sim 120 \mathrm{pg} / \mathrm{ml}$, is linear to $9500 \mathrm{pg} / \mathrm{ml}$, is stereo-specific, and is minimally cross-reactive to other eicosanoids.

Growth cone collapse assay. DRG cultures were preincubated (at $37^{\circ} \mathrm{C}$ ) with inhibitor or vehicle control (DMSO or ethanol) for 45-60 min before they were inverted onto media-filled Coverwell (Grace Bio-Labs) imaging chambers. The imaging chambers were then placed onto the heated stage $\left(37^{\circ} \mathrm{C}\right)$ of a Zeiss Axiophot microscope and allowed to equilibrate for $15 \mathrm{~min}$ before repellent factor treatment. Images were captured at $t=-5,0,1,2.5,5,7.5,10$, and $15 \mathrm{~min}$ after the addition of repellent using a Kodak digital camera. Growth cone collapse was assessed quantitatively by recording the area of growth cone spread before and after treatment (using NIH image 1.62 software). The results were expressed as the percentage change in growth cone area. Student's $t$ test was used to assess the statistical significance of experimental results.

Interference reflection microscopy. Coverslips with DRG cultures were mounted onto viewing chambers in medium, covered with mineral oil, and analyzed on a heated stage with an appropriately equipped Zeiss Axiovert 200M microscope. Images were analyzed by NIH image 1.62 . The density slice function was used to isolate the darkest areas of a growth cone image. Total area of that region was measured. Student's $t$ test was used to assess the statistical significance of experimental results.

Fluorescence imaging. After experimentation, cultures were fixed by slow infusion of $4 \%$ paraformaldehyde in $0.1 \mathrm{~m}$ phosphate buffer, $\mathrm{pH} 7.4$, into the culture dishes over 10 min (Pfenninger and Maylie-Pfenninger, 1981). Thereafter, the fixative was removed by rinsing three times with PBS containing $1 \mathrm{~mm}$ glycine. Cultures were blocked with $\mathrm{PBS} / 1 \%$ bovine serum albumin (BSA) and permeabilized by incubation in $0.02 \%$ Triton X-100 for 5 min. After three washes in PBS/BSA, cultures were ready for staining.

To stain for F-actin, cultures were incubated with Texas-Redconjugated phalloidin for $30 \mathrm{~min}$. Unbound phalloidin was washed away by two PBS rinses, after which the cover glasses were mounted in anti-FADE reagent (Molecular Probes, Eugene, OR). Paxillin and NP-1 were visualized by incubating the cultures with either anti-paxillin antibody (Transduction Laboratories, Lexington, KY) or anti-NP-1 antibody 1:100 in PBS/BSA at room temperature for $1 \mathrm{hr}$. Cultures were washed again and incubated for 45 min with either Texas-Red-conjugated goat anti-rabbit IgG for NP-1 (1:100; Molecular Probes) or Oregon-Greenconjugated goat anti-mouse IgG (1:100; Molecular Probes) and TexasRed-conjugated phalloidin (5 U/ml; Molecular Probes) for dually labeled samples. After three washes, the coverslips were mounted onto slides in anti-FADE reagent.

Coverslips were examined with a Zeiss Axiophot fluorescence microscope equipped with epi-illumination. Confocal images were captured using an Olympus IX70 inverted microscope equipped with a Photometrics PXL camera $(67 \mathrm{~nm}$ per image pixel with a $100 \times$ oil immersion
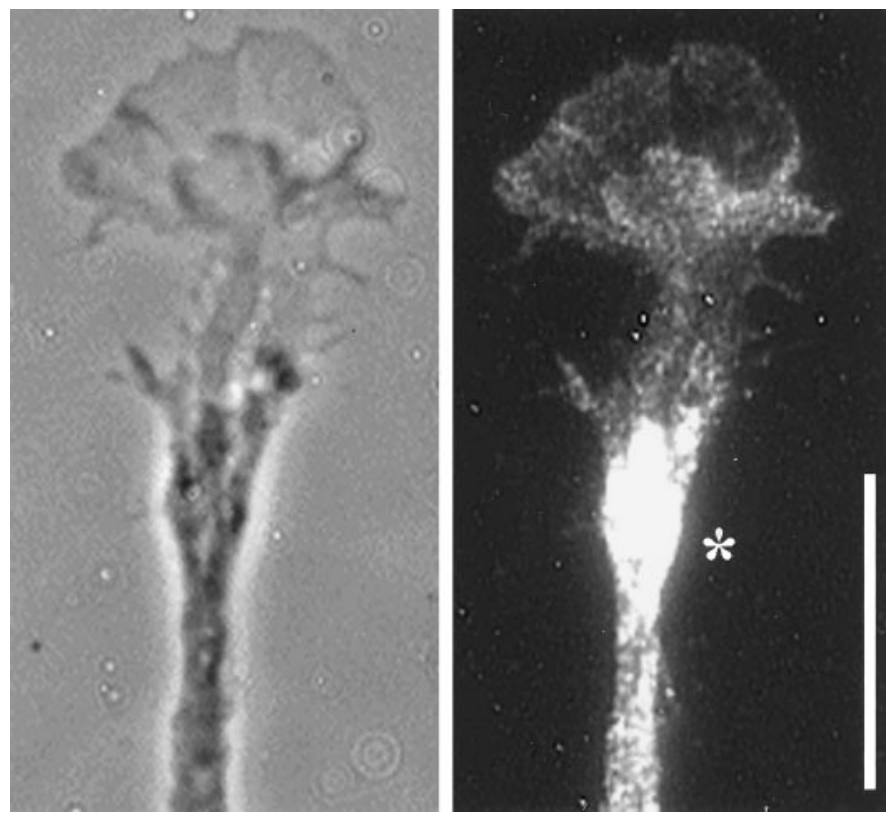

Figure 1. Immunolocalization of NP-1 in NGF-responsive growth cones of DRG neurons. Left panel, Phase-contrast image shown for comparison. Right panel, Immunofluorescence image of bound anti-NP-1. Nonspecific fluorescence in controls processed without primary antibody was below the level of detection. Anti-NP-1 generates a diffuse, punctate pattern of immunoreactivity that is detected throughout the neurite and growth cone and extends into the finest filopodia. The asterisk indicates an area of high fluorescence intensity discussed in Results. Scale bar, $15 \mu \mathrm{m}$.

objective) and a Silicon Graphics $\mathrm{O}_{2}$ computer with DeltaVision deconvolution software (Applied Precision).

\section{RESULTS}

\section{Neuropilin-1 is distributed throughout the growth cone}

Indirect immunofluorescence was performed on cultures of primary DRG neurons to determine the prevalence of NP-1-positive neurites in our preparations and to assess morphologically the distribution of NP-1 in their growth cones. Specific NP-1 fluorescence was observed in all neurons and neurites and thus confirmed that our DRG cultures are a valid system for studying quantitatively the growth cone responses to Sema3A (Fig. 1). Controls that omitted the primary antibody were free of fluorescence (data not shown).

Detailed examination of the growth cones revealed punctate NP-1 staining. Labeling extended, at a moderate level, from the proximal domain into the thinnest regions of the growth cone periphery and into the finest filopodia. The staining in these distal regions appeared uniformly punctate. Robust labeling of neurite bundles indicated the presence of NP-1 in neurite shafts. Whether all NP-1 was on the cell surface, and whether its level was higher on neurites than on growth cones, however, was not evident because the optical superimposition of images generated by neurite fascicles contained much more plasma membrane than those of the growth cone periphery. In addition, cytoplasmic membrane elements were present. Areas of intense fluorescence (Fig. 1, asterisk) were observed occasionally in preterminal areas of fasciculated neurites. These may represent accumulations of NP-1-rich precursor membrane waiting to be inserted into the growth cone plasmalemma, they may be the result of NP-1 internalization, or they may serve some other, unknown function. 


\begin{tabular}{lll}
\hline $\begin{array}{l}\text { Table 1. Sema3A stimulates 12(S)-HETE generation in sprouting } \\
\text { DRG neurons }\end{array}$ & \\
& $\begin{array}{l}\text { Concentration } \\
\text { (CDC/Sema3A) }\end{array}$ & $\begin{array}{l}\text { 12(S)-HETE (femtomoles } \\
\text { of HETE per microgram } \\
\text { of proin) }\end{array}$ \\
Treatment & $-/-$ & $11.1 \pm 0.01$ \\
Untreated & $-/ 1 \mathrm{nM}$ & $97.5 \pm 18$ \\
Sema3A & $10 \mu \mathrm{m} / 1 \mathrm{nM}$ & $4.8 \pm 0.04$ \\
CDC + Sema3A & $10 \mu \mathrm{M} /-$ & Not detectable \\
CDC &
\end{tabular}

12(S)-HETE levels of cell lysates obtained after indicated experimental treatments were measured using an immunobinding assay. Data are from a representative experiment done in duplicate (values are means \pm SD). All measurements were obtained in the same experiment.

\section{Eicosanoid generation in DRG neurons}

We know that thrombin-treated cortical neurons and isolated growth cones generate 12(S)-HETE and that 12(S)-HETE is necessary for growth cone collapse in that system (de la Houssaye et al., 1999). To test our current hypotheses, we first examined whether Sema3A stimulated the synthesis of 12(S)-HETE. 12(S)HETE levels were determined in sprouting DRG cultures using an immunobinding assay. Resting 12(S)-HETE levels were $\sim 11$ $\mathrm{fmol} / \mu \mathrm{g}$ protein (Table 1 ). This value is based on total protein in the cultures, rather than just growth cone protein. Because cPLA and 12/15-LO activities are enriched in growth cones (NegreAminou and Pfenninger, 1993; de la Houssaye et al., 1999), the actual level of 12(S)-HETE in DRG growth cones is likely to be higher. In response to Sema3A treatment for $2 \mathrm{~min}$, the level of 12(S)-HETE increased up to approximately ninefold (Table 1). The limited number of DRG cultures that could be prepared in a single session did not allow for the generation of a dose-response curve. However, in two separate experiments an increase in
Sema3A concentration from $\sim 0.5$ to $\sim 1$ nM raised the level of 12(S)-HETE detected 4.9- and 2.7-fold (3.8-fold average). In parallel experiments, the same increase in Sema3A concentration caused a 4.1-fold enhancement in collapse (area decrease, see below). Thus, Sema3A-stimulated 12(S)-HETE synthesis was dose dependent and paralleled the collapse response, at least within a limited concentration range. The Sema3A-stimulated increase in 12(S)-HETE was reduced to below resting level by preincubating the cultures with CDC for $45 \mathrm{~min}$ before exposure to Sema3A. In the micromolar range, $\mathrm{CDC}$ is a selective inhibitor of LOs, including 12/15-LO (Cho et al., 1991). In cultures that were treated with CDC only, 12(S)-HETE was not detectable.

\section{Is 12/15-LO necessary for Sema3A-induced collapse?}

To answer this question we performed quantitative collapse assays on growth cones of rat DRG neurons in culture. Figure 2 shows in representative phase-contrast micrographs the effects of the experimental treatments on growth cone morphology at the beginning $(t=0)$ and end of a 7.5 min challenge with a subsaturating concentration $(\sim 0.5-1.0 \mathrm{~nm})$ of Sema3A. Quantitation of growth cone collapse was accomplished by measuring total area of the same live growth cones, at the beginning and after $7.5 \mathrm{~min}$ of experimental treatment. This was done for 25 growth cones per experimental condition. Measurement of the same growth cones in this before/after paradigm had the advantage of determining actual change in area independent of the highly variable starting configuration of the growth cone. Quantitative results were expressed as the percentage change in growth cone area measured over the experimental time period and are shown in Figure 3.

Within 3 min of Sema3A treatment, many growth cones lost their lamellipodial veils (data not shown). Treatment for $7.5 \mathrm{~min}$ (Fig. 2) caused the expected collapse, with a highly significant reduction of $>30 \%$ of growth cone area (Fig. 3) $\left(p<10^{-4}\right)$ and

Figure 2. Effects of Sema3A, of an LO inhibitor (CDC), of $\mathrm{LO}$ inhibitor plus Sema3A, and of LO inhibitor plus 12(S)HETE on growth cone morphology. Phase-contrast micrographs show growth cones in cultures of rat DRG neurons that were either pretreated with vehicle alone or with CDC for 45 min before challenge with Sema3A or 12(S)-HETE. Images in the top row were taken at time 0 ; those in the bottom row were taken after $7.5 \mathrm{~min}$ of challenge with collapsing agent. Left to right, $10 \mu \mathrm{M}$ CDC pretreatment, $10 \mu \mathrm{M}$ CDC pretreatment followed by Sema3A $(\sim 0.5 \mathrm{nM})$, Sema3A $(\sim 0.5 \mathrm{nM})$, and $10 \mu \mathrm{M}$ CDC pretreatment followed by $12(\mathrm{~S})$ HETE (100 nM). Arrows point to growth cones that are collapsing. Scale bar, $20 \mu \mathrm{m}$.

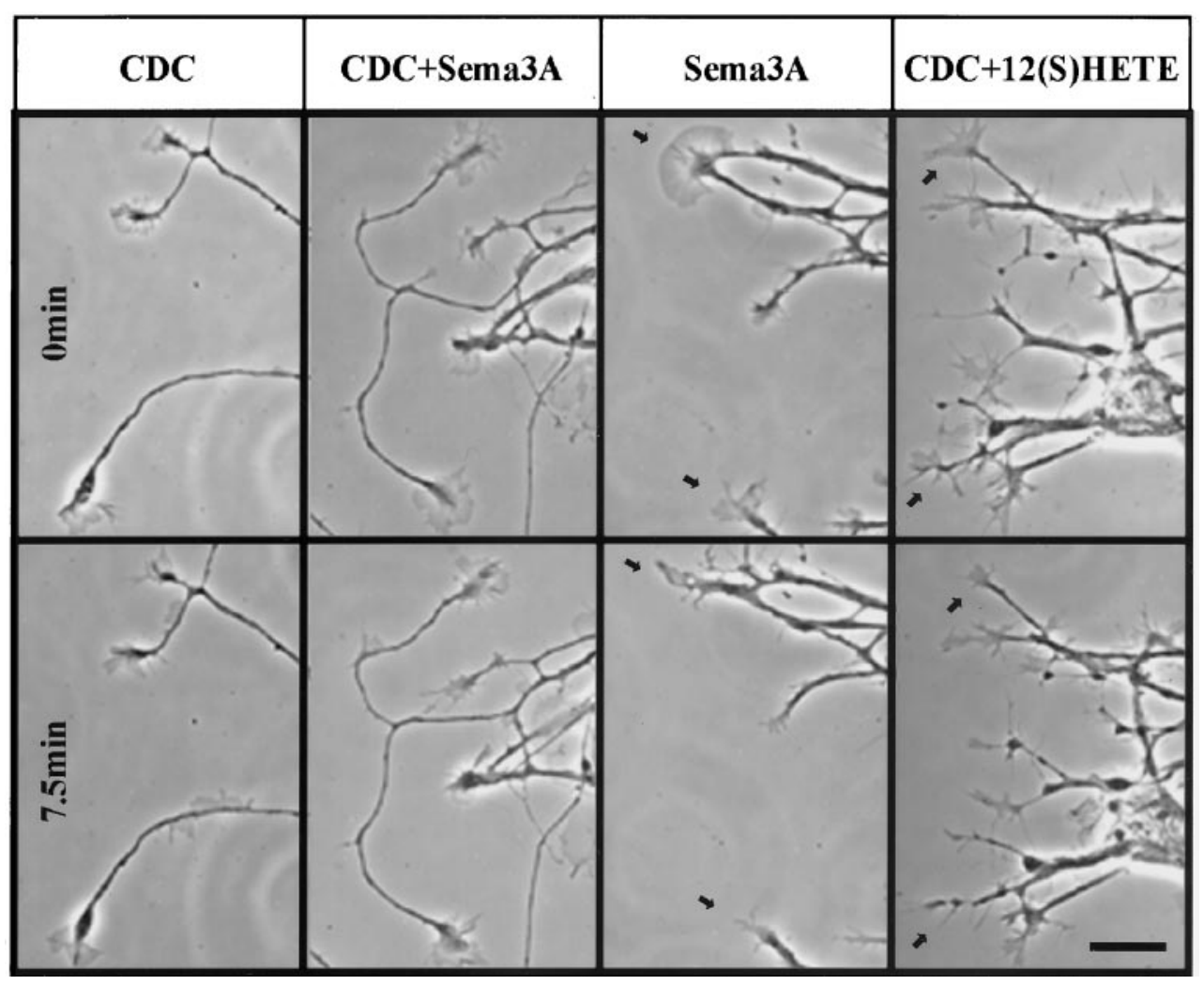




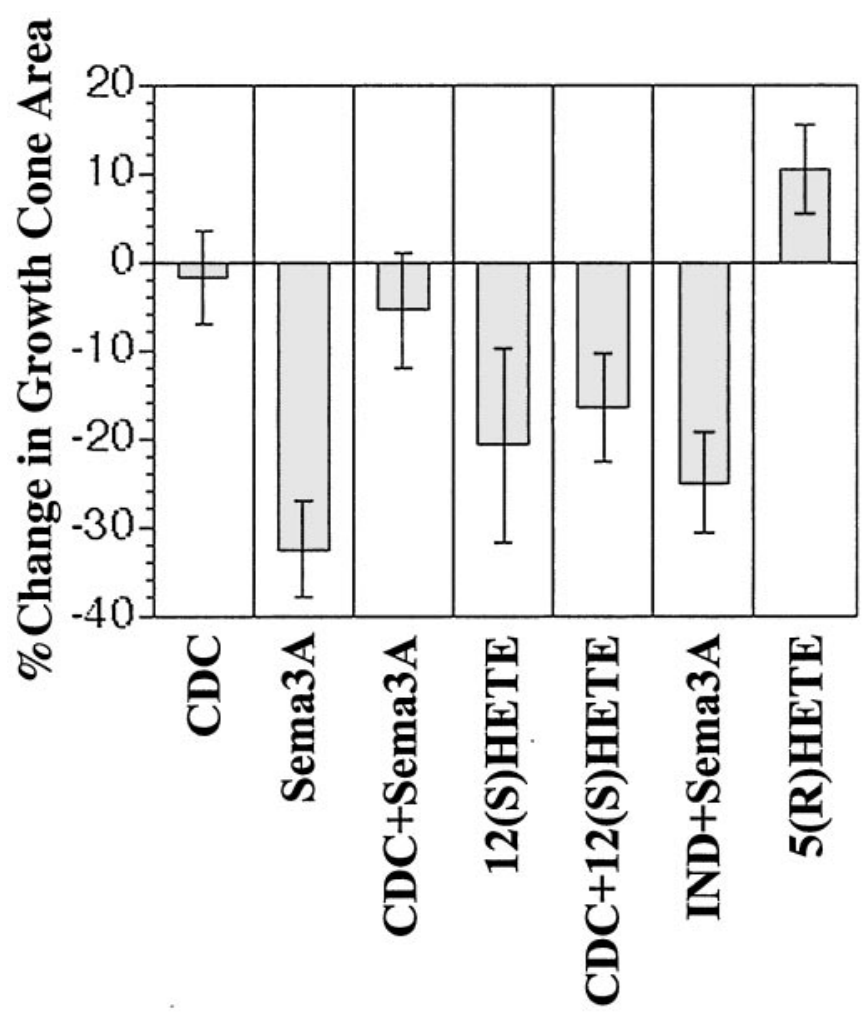

Figure 3. Quantitative analysis of collapse measured as percentage of change in growth cone area after $7.5 \mathrm{~min}$ of experimental treatment \pm SEM. Twenty-five growth cones were measured for each condition over a minimum of three independent experiments. Left to right: $10 \mu \mathrm{M} \mathrm{CDC}$ pretreatment; Sema $3 \mathrm{~A}(\sim 0.5 \mathrm{nM}) ; 10 \mu \mathrm{M}$ CDC pretreatment followed by Sema3A ( 0.5 nM); 12(S)-HETE (100 nM); $10 \mu \mathrm{M}$ CDC pretreatment followed by 12(S)-HETE (100 nM); $10 \mu \mathrm{M}$ indomethacin pretreatment followed by Sema3A ( $\sim 0.5 \mathrm{nM})$; and $5(\mathrm{R})$-HETE.

concomitant loss of filopodia and lamellipodia. Increasing the Sema3A concentration enhanced the collapse response to reach a saturating level at $\sim 75 \%$ of area reduction (data not shown). This resulted often in neurites terminating in a "club"-shaped structure that sometimes possessed a few filopodia. In contrast to thrombin and lysophosphatidic acid-induced collapse (Jalink et al., 1993; de la Houssaye et al., 1999), little or no neurite retraction was observed for Sema3A in this time frame.

To determine whether signaling involved in Sema3A-induced growth cone collapse requires 12/15-LO activity, we preincubated DRG neurons with CDC, a potent and selective LO inhibitor. As a control we used indomethacin, a specific inhibitor of cyclooxygenases, thereby blocking the synthesis of prostaglandins and related eicosanoids, but not of HETEs. The growth cones of cultures pretreated with only CDC and observed over time (up to $2 \mathrm{hr}$ ) were fully spread out and veiled with extensive lamellipodia and filopodia (Fig. 2, $C D C$ ). By our current criteria they were indistinguishable from untreated cultures.

In cultures pretreated with CDC, Sema3A exposure for $7.5 \mathrm{~min}$ (Figs. 2, 3, $C D C+S E M A 3 A$ ) failed to induce growth cone collapse. These growth cones retained their lamellipodia and filopodia and, in some cases, continued to advance. Quantitatively growth cone areas remained indistinguishable (two-tailed $t$ test) from those in CDC-only or control cultures. In contrast, if cultures were pretreated with indomethacin before challenge with Sema3A (Fig. 3, IND + Sema3A), growth cones collapsed, losing $\sim 25 \%$ of their area, essentially as though they had not been pretreated.

\section{Is the 12/15-LO product, 12(S)-HETE, sufficient to induce growth cone collapse?}

To examine whether the products of 12/15-LO are sufficient for growth cone collapse, we applied exogenous 12(S)-HETE to cultures that had or had not been preincubated with CDC (Figs. 2, 3, CDC +12(S)-HETE, 12(S)-HETE). These experiments revealed that $12(\mathrm{~S})$-HETE caused collapse alone and even in the presence of CDC when 12/15-LO was inhibited. In both cases, 12(S)-HETE-treated growth cones gradually withdrew their lamellipodia until they lost both lamellipodia and filopodia. After $7.5 \mathrm{~min}$ of $12(\mathrm{~S})$-HETE treatment, growth cones exhibited a significant loss of $\sim 20 \%$ of total area (Fig. 3) regardless of whether they had been pretreated with CDC $(p<0.06$ for HETE alone; $p<0.04$ for CDC + HETE). This degree of area reduction was more modest than that achieved by Sema3A but nevertheless significant. To rule out possible nonspecific effects that may be induced by the eicosanoid treatment of our cultures, we also tested a regio-isomer, 5(R)-HETE. Interestingly, the addition of $5(\mathrm{R})$-HETE to cultures caused an increase $(\sim 10 \%)$, rather than a decrease, in growth cone area (Fig. 3), which indicated regio-isomer specificity of the HETE effects. These data demonstrate that $12 / 15-\mathrm{LO}$ is necessary for Sema3Ainduced growth cone collapse and that 12(S)-HETE, but not 5(R)-HETE, is sufficient to induce collapse.

\section{Effects of Sema3A and HETE on adhesion sites}

Growth cone collapse must occur as a result of at least two mechanisms: loss of F-actin from the growth cone periphery and disruption of growth cone adhesion sites (de la Houssaye et al., 1999; Zhou and Cohan, 2001). To test directly whether 12(S)HETE caused alteration of adhesion sites, we used interference reflection microscopy (IRM), which allows the investigator to view, in live cells, changes in cellular adhesion sites. IRM images areas of close apposition of the plasma membrane to the glass coverslip $(<10 \mathrm{~nm})$ as very dark; areas of more open cellular apposition appear lighter than background (Izzard and Lochner, 1976).

Figure 4 shows representative IRM images taken of growth cones before and after $7.5 \mathrm{~min}$ of challenge. At the onset of challenge, growth cones exhibited a variable but primarily punctate and somewhat radial pattern of close (dark) adhesions with occasional bright (intermediate) adhesion areas (Fig. 4, left panels). After Sema3A or 12(S)-HETE challenge, the growth cone contact area was reduced greatly. Some close contact areas were changed to bright (Sema3A, arrowhead), indicating loosened adhesion. To quantify these changes, growth cone images were processed digitally by thresholding to isolate and measure the dark adhesion sites (Fig. $5 A$ ). Over the 7.5 min experimental time period, dark areas of control growth cones did not change significantly $(-8 \pm 6 \%$; mean \pm SEM). In contrast, Sema3A or 12(S)-HETE treatment of the cultures decreased the dark regions by $\sim 45 \%$, a value that is greater than the growth cone area decrease reported above (Fig. 3). This provides direct evidence that Sema3A and exogenous 12(S)-HETE rapidly cause at least partial dissociation of adhesion sites in growth cones. Quantitatively, i.e., for the population of growth cones analyzed, CDC pretreatment plus Sema3A challenge did not reduce close contact area. Of particular interest was the appearance of a prominent, almost continuous distal band of attachment. Instead of the primarily radial pattern of dark areas at $0 \mathrm{~min}$, we observed an 

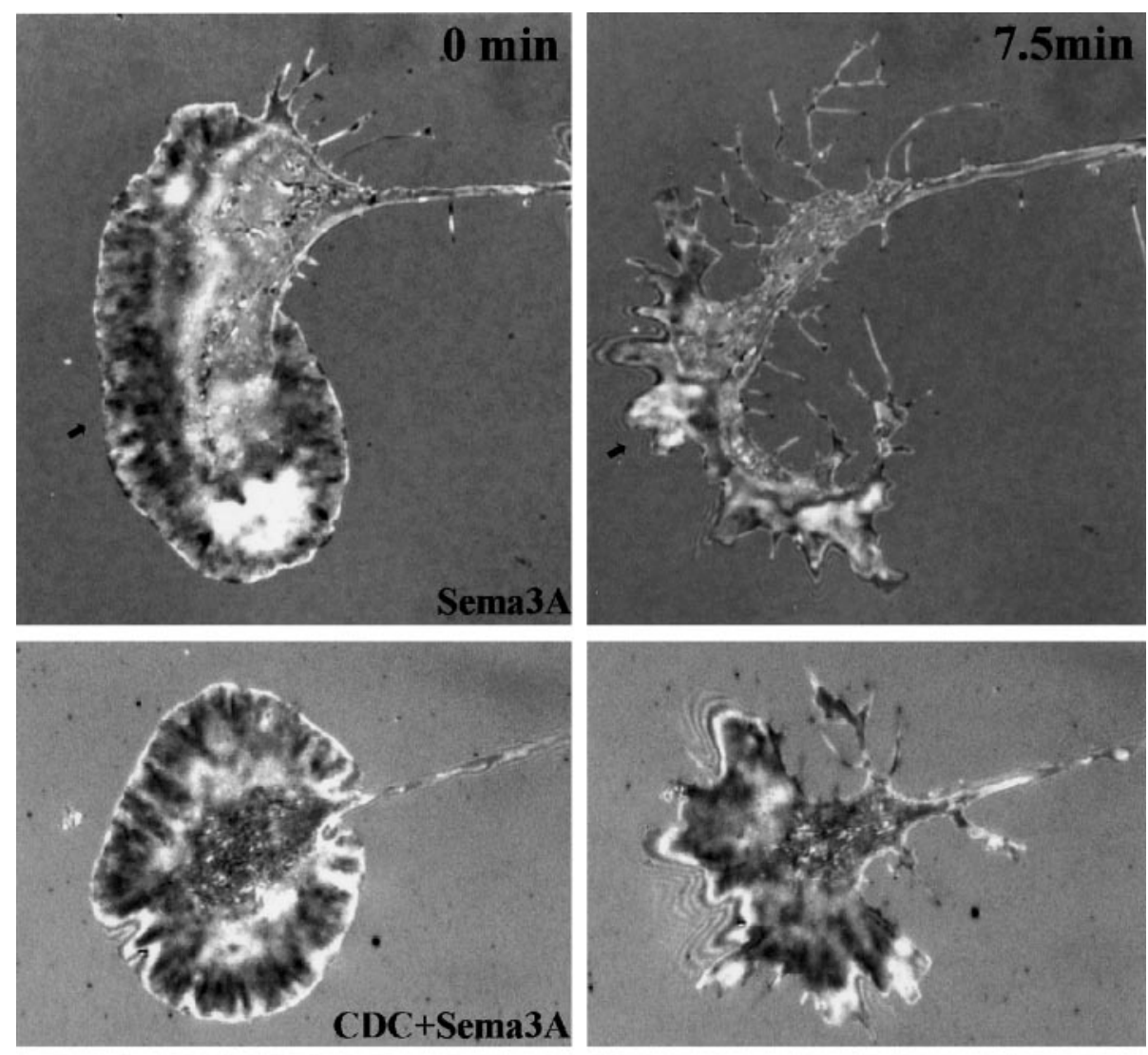

Figure 4. Sema3A and 12(S)-HETE treatment of DRG growth cones causes a loss of adhesive area. IRM images were taken before (left) and after (right) $7.5 \mathrm{~min}$ of treatment with (top to bottom) Sema3A ( 1 nM), Sema3A after $30 \mathrm{~min}$ CDC $(10 \mu \mathrm{M})$, or 12(S)-HETE (100 nM). Arrows point at an area of conversion from predominantly dark (close contact) to bright (intermediate contact).
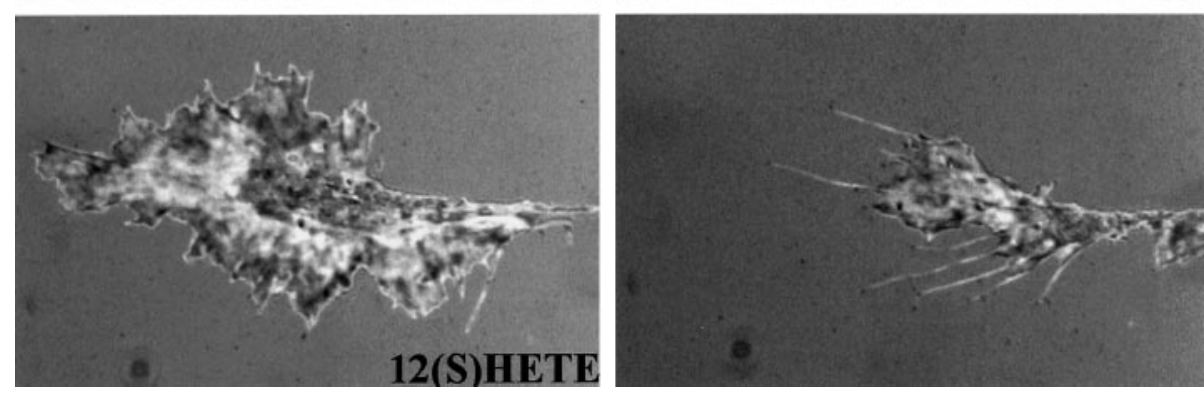

IRM-dark peripheral band adjacent to a more distal IRM-bright rim of close and intermediate attachment, respectively (Fig. 3, $C D C+\operatorname{Sema3A}, 7.5 \mathrm{~min})$. Although IRM patterns of thin structures must be interpreted with caution, a marginal band of adhesion was evident.

With a complementary approach we examined how both the actin cytoskeleton and adhesion sites were affected by our experimental treatments. We fixed cultures after challenge and then stained them with fluorescent labels for F-actin and the adhesion site protein, paxillin. In previous studies paxillin has proven to be an excellent marker of adhesion sites (Ross et al., 2000). Figures 6 and 7 show the confocal images of the attachment areas of the growth cones. CDC-treated (Fig. 6, CDC) growth cones exhibited broadly spread lamellipodia with F-actin-positive radial ridges that originated near the neurite shaft and extended to the distal edge of the growth cone. The area of greatest label intensity was near or at the distal edge. Paxillin staining in these cultures appeared as a partially overlapping, punctate pattern. It was particularly dense in the neurite and central region of the growth cone but extended all the way into the periphery. The function of the high paxillin level in the neurite is unknown. Relative to the other peripheral areas of the growth cone, the paxillin puncta seemed to be slightly more concentrated along the distal edge and the radial ridges highlighted by F-actin. This observation is consistent with a requirement for distal adhesion sites in the spread growth cone.

After $7.5 \mathrm{~min}$ of Sema3A treatment (Fig. 7, Sema3A), growth cones had lost their lamellipodia and all but a few of their filopodia, with the actin cytoskeleton being collapsed to a proximal, "clumped" pattern, where it colocalized with paxillin. This is consistent with what others have observed (Fournier et al., 2000b). Interestingly, we observed a very similar response in thrombin-treated cortical neurons (de la Houssaye et al., 1999), which supports the idea that proximal "clumping" of actin may represent a universal feature of growth cone collapse.

We also examined cultures that were pretreated with $\mathrm{CDC}$ before Sema3A challenge. In most cases (Fig. 6, $C D C+S e m a 3 A$ ), the growth cones remained spread out and retained their lamellipodia despite having lost their radial actin ridges. Fluorescent intensity for both paxillin and F-actin was strongest along the distal edge of the growth cone, where they colocalized (yellow). This yellow band seemed to correspond to the peripheral band of 

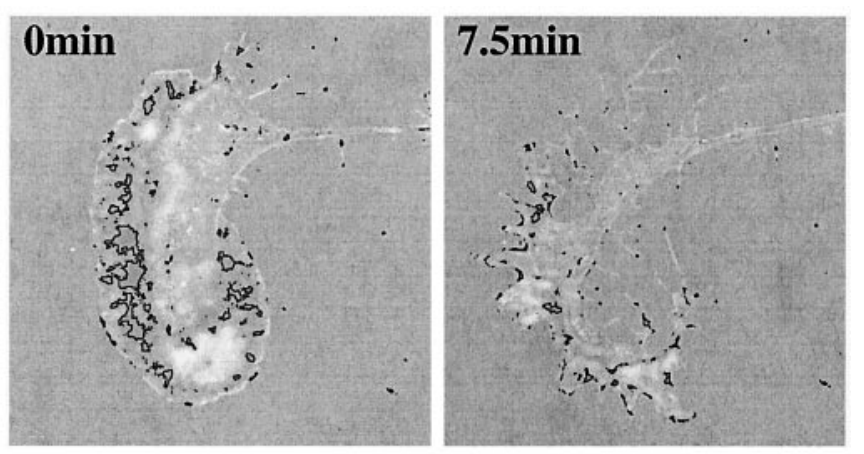

A

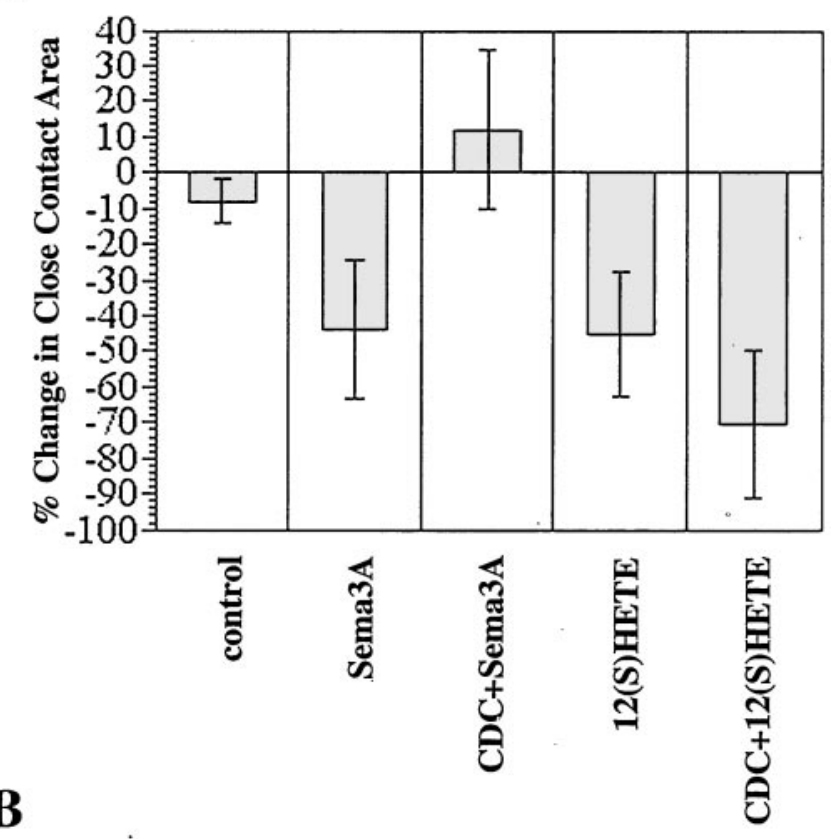

Figure 5. Quantitative analysis of the decrease in IRM-dark areas under various experimental conditions. $A$, Density slices of a growth cone before and after treatment $(7.5 \mathrm{~min})$ with Sema3A $(\sim 1 \mathrm{nM})$. These were derived from the top panels of Figure 4. B, Changes in the IRM-dark areas (close contacts) of growth cones treated with Sema3A $(\sim 1 \mathrm{nM})$ or $12(\mathrm{~S})$-HETE (100 $\mathrm{nM})$ for $7.5 \mathrm{~min}$, without or with CDC preincubation (30 min). control, No treatment. Data are averages \pm SEM $(n \geq 5$, except for CDC + Sema3A, where $n=2$ ).

attachments observed by IRM in the same type of experiment (Fig. 4). This led us to suspect that the adhesion sites remained intact, especially along the distal edge, notwithstanding the compromised structural integrity associated with loss of distal F-actin. Some degree of proximal F-actin clumping also was evident in these growth cones.

Growth cones of DRG neurons treated with 12(S)-HETE lost a significant fraction of their spread area and close contacts (Figs. $2,3,4)$. However, in growth cones that were not yet fully collapsed (Fig. 7, 12(S)-HETE), we observed that a significant amount of F-actin remained distributed in the growth cone periphery, chiefly in filopodia, with some actin ridges still evident (see corresponding single channel image). Little or no actin clumping was observed proximally in these cases. Paxillin was distributed in a somewhat more condensed punctate pattern, extensively colocalized with F-actin. The paxillin-enhanced distal edge of the growth cone, characteristic of $\mathrm{CDC}+\mathrm{Sema3A}$ experiments, was not evident.

\section{DISCUSSION}

Sema3A triggers growth cone collapse in neurons expressing NP-1/plexin A receptors (Goshima et al., 1999; Reza et al., 1999; Takahashi et al., 1999), but the identification of the signaling steps leading to collapse has remained elusive. We report that Sema3A increases 12(S)-HETE levels in DRG neurons and that this eicosanoid is necessary for growth cone collapse. We also show that $12(\mathrm{~S})$-HETE is sufficient to cause collapse and that it does so by reducing growth cone adhesion.

\section{Role of eicosanoid in Sema-induced collapse}

Our cultured DRG neurons exhibited a uniform population of NP-1 receptor-positive growth cones. This enabled us to investigate the role of $12 / 15-\mathrm{LO}$ in Sema3A signaling. Cellular eicosanoid generation depends on the supply of arachidonic acid, a $\mathrm{PLA}_{2}$ cleavage product. Indeed, the collapsing factor, thrombin, greatly stimulates $\mathrm{PLA}_{2}$ activity in isolated growth cones (de la Houssaye et al., 1999). However, PLA 2 is such a widespread biological activity that its changes (e.g., triggered by Sema3A) in a subset of cellular elements (e.g., growth cones in DRG cultures) are difficult to detect against background levels. The next step in 12(S)-HETE synthesis, catalyzed by $12 / 15$-LO, appears to be more specific. The recent introduction of a highly sensitive immunobinding assay for 12(S)-HETE has made it possible to measure Sema3A-induced changes in the level of this eicosanoid.

When exposed to Sema3A, DRG cultures increased the level of 12(S)-HETE several-fold. This increase was comparable to that observed for thrombin-treated, isolated growth cones (de la Houssaye et al., 1999). In these earlier growth cone studies, we measured rates of eicosanoid synthesis from exogenous radiolabeled substrate. Because those data are not directly comparable to the actual eicosanoid levels in DRG cultures shown here, we analyzed isolated growth cones with both assays in parallel, using thrombin as the repellent factor. Our results (data not shown) suggest that the 12(S)-HETE accumulation expected from the measured, thrombin-stimulated increase in eicosanoid synthesis [radiolabel assay; see de la Houssaye et al. (1999)] is comparable to the actual increase in 12(S)-HETE as determined by immunobinding. Also of importance, at least within a limited range of Sema3A concentrations, growth cone collapse and 12(S)-HETE generation increase roughly in parallel, suggesting that $12 / 15-\mathrm{LO}$ may play a role in Sema3A-induced growth cone collapse, as in thrombin signaling.

To test this hypothesis we inhibited 12/15-LO activity by pretreatment of the cultures with CDC, a selective blocker of LOs (Cho et al., 1991). Biochemical measurements showed that CDC decreased even Sema3A-stimulated 12(S)-HETE levels to below control. In these conditions, Sema3A failed to cause growth cone collapse. In contrast, the cyclooxygenase inhibitor, indomethacin, did not interfere with collapse triggered by this repellent. These results indicated that $12 / 15-\mathrm{LO}$ is necessary for Sema3A-induced collapse. To assess whether 12(S)-HETE is sufficient to cause collapse, we applied exogenous 12(S)-HETE to DRG growth cones, with or without previous CDC incubation. As in the case of cortical neurons (de la Houssaye et al., 1999), growth cones of DRG neurons collapsed in response to 12(S)-HETE, regardless of the presence of CDC. The result indicates that this eicosanoid can reverse the CDC inhibition of collapse and that it is sufficient to cause collapse. Interestingly a regio-isomer, 5(R)-HETE, increased rather than decreased growth cone spread by a small, but 

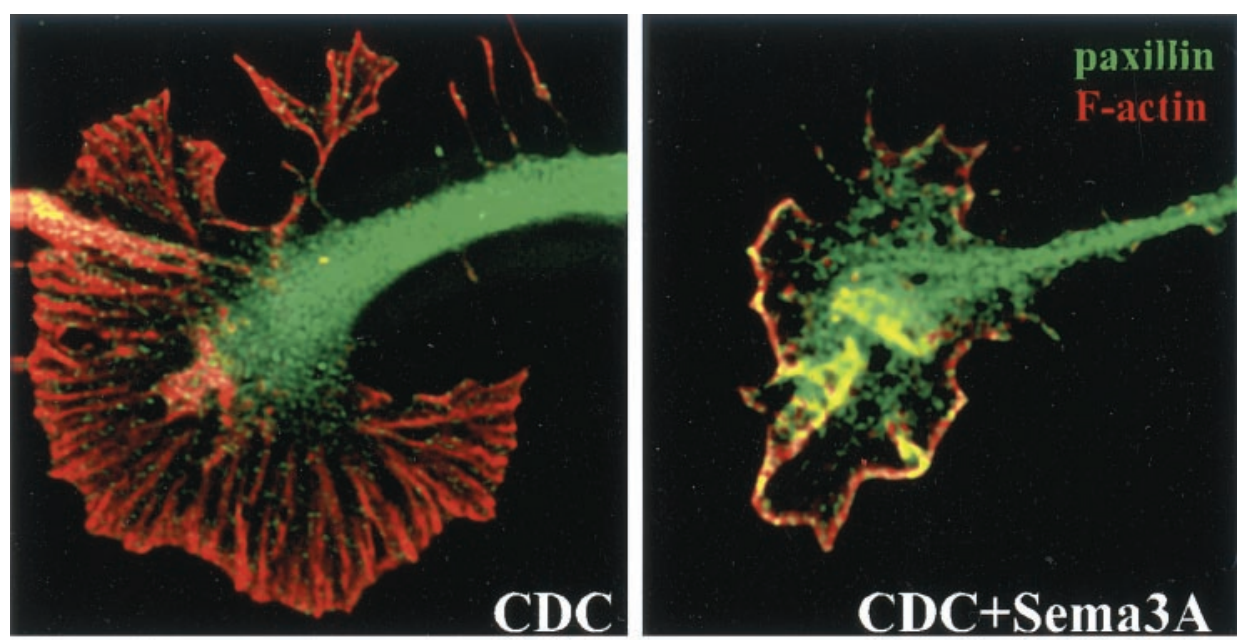

Figure 6. Effects of CDC and Sema3A on growth cone F-actin and paxillin as shown by confocal microscopy. Images are optical sections through the attachment area. DRG growth cones were pretreated with $10 \mu \mathrm{M}$ CDC for $45 \mathrm{~min}$ before challenge $(7.5 \mathrm{~min})$ with control medium or Sema3A $(\sim 0.5 \mathrm{~nm})$. Collapse effects paralleled those reported in Figure 3. F-actin is shown in red, paxillin is shown in green, and areas of overlap appear yellow. From top to bottom, panels show merged images, F-actin only, and paxillin only. Scale bar, $15 \mu \mathrm{m}$.
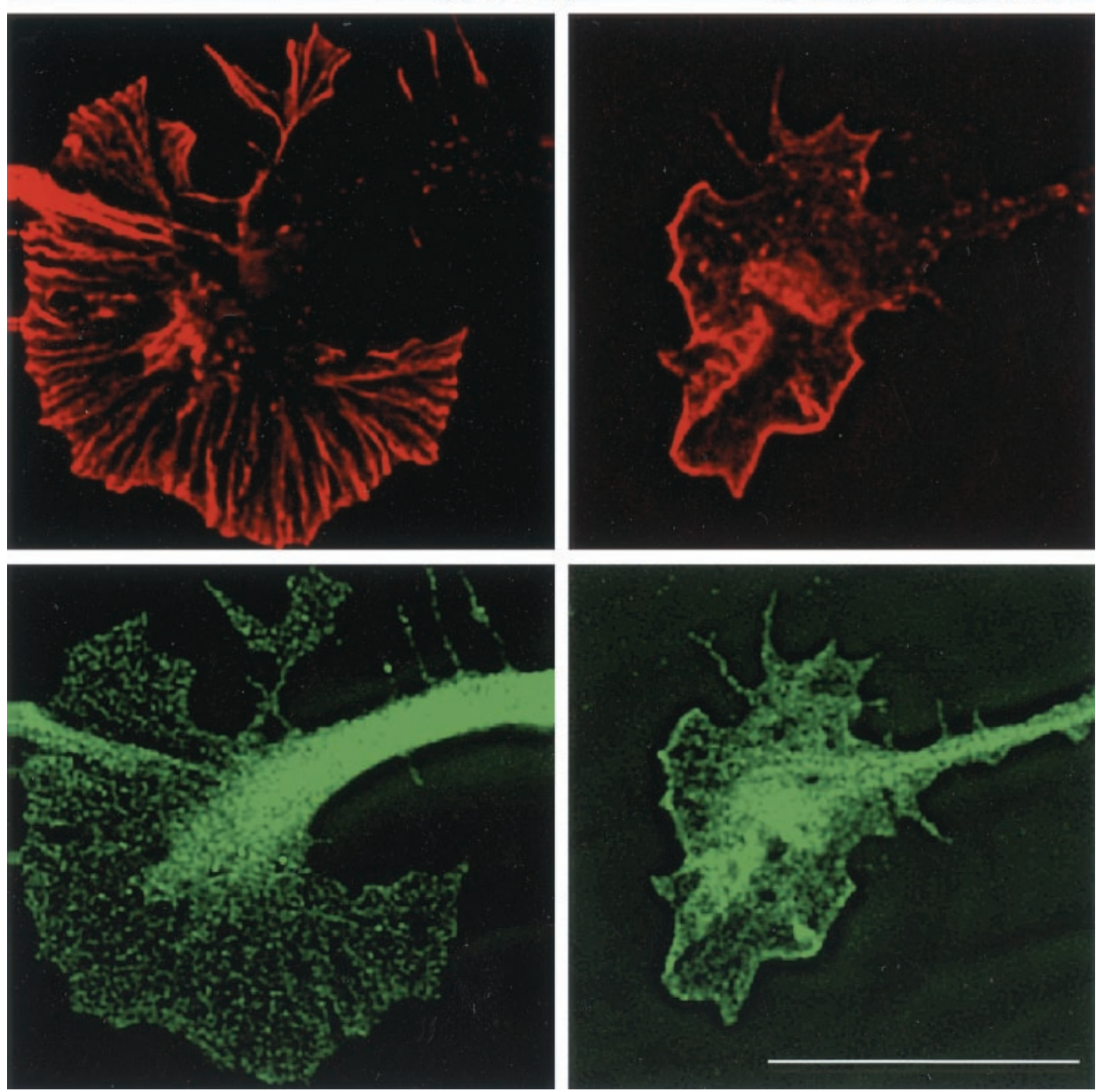

significant, amount. Therefore, the 12(S)-HETE-induced collapse is a specific effect of this isomer.

\section{Eicosanoids and growth cone adhesion}

Our earlier experiments with isolated growth cones demonstrated that thrombin or thrombin receptor-activating peptide causes growth cone detachment from the substratum independent of the actin cytoskeleton. Because experiments involving isolated growth cones are not feasible for DRG neurons, we examined their attachment to the substratum by IRM. Within minutes, Sema3A and exogenous 12(S)-HETE were seen to reduce areas of both intermediate and close contacts. Close-contact areas were shown to change significantly, and this reduction in contact was greater than the decrease in growth cone area observed under the same conditions. However, CDC inhibited the Sema3A-induced reduction in contact area. These observations indicate that $12(\mathrm{~S})$ HETE diminishes growth cone adhesion.

To study changes in growth cone adhesion in further detail, we analyzed growth cone collapse by confocal microscopy, using paxillin antibody and phalloidin to reveal adhesion sites and F-actin, respectively. The observed high level of paxillin in the neurite shaft is surprising and difficult to explain at the present. The images of control (or CDC pretreated) and of 

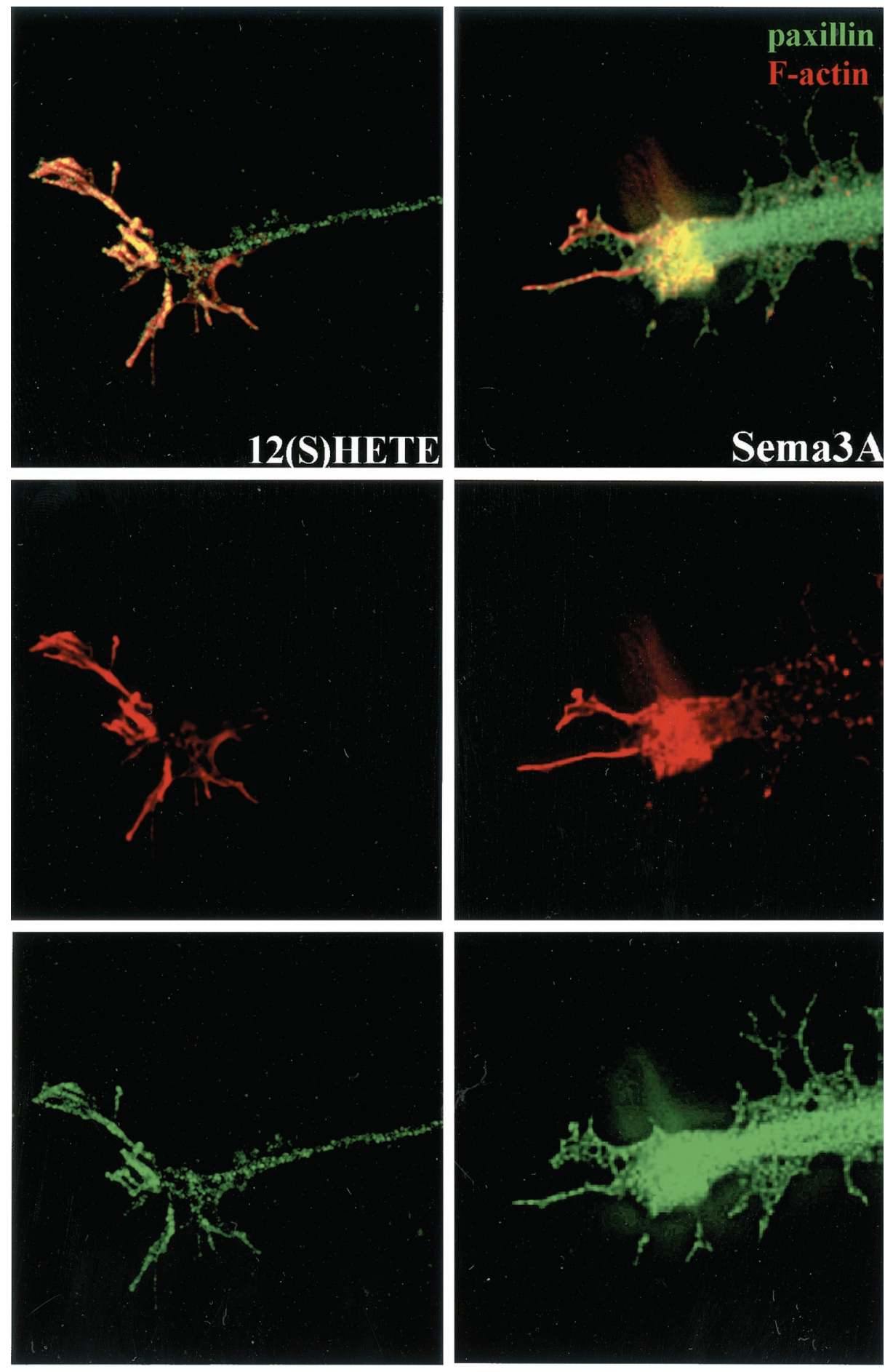

Figure 7. Effects of Sema3A and 12(S)-HETE on growth cone F-actin and paxillin as shown by confocal microscopy. Images are optical sections through the attachment area. DRG growth cones were challenged with Sema3A $(\sim 0.5 \mathrm{~nm})$ or 12 (S)-HETE (100 nM). Collapse effects paralleled those reported in Figure 3. To illustrate the relative changes in actin and paxillin distribution, however, the growth cone example for 12(S)-HETE (left) is one of incomplete collapse. F-actin is shown in red, paxillin is shown in green, and areas of overlap appear yellow. From top to bottom, panels show merged images, F-actin only, and paxillin only. Scale bar (shown in Fig. 6), $15 \mu \mathrm{m}$.
Sema3A-collapsed growth cones were consistent with earlier reports (Fournier et al., 2000b). Most striking was the result of the $\mathrm{CDC}+$ Sema3A experiment: growth cones remained spread out and exhibited a distal, continuously labeled rim of paxillin as if the adhesion sites had consolidated into an adherent margin. The usually radial F-actin pattern had disappeared, but a distal rim of actin, colocalized with paxillin, persisted. Corresponding IRM images did indeed show such a distal rim of attachment. In other words, growth cones remained spread and attached by a peripheral band of paxillinand F-actin-containing adhesions despite Sema3A-induced F- actin loss more proximally. This result is consistent with what we observed in previous studies in which CDC + thrombintreated growth cones of cortical neurons remained spread, although the majority of F-actin was lost (de la Houssaye et al., 1999). Therefore, inhibition of 12/15-LO does not appear to block F-actin loss within the time frame of these observations.

In contrast to the previous experiment, the paxillin- and F-actin-containing rim seen after CDC + Sema3A was not evident in growth cones treated with 12(S)-HETE or with CDC + 12(S)-HETE (data not shown). The growth cones of 12(S)HETE-treated neurons collapsed, although F-actin retained a 
rudimentary radial pattern. This would be expected initially, if an otherwise intact growth cone were to lose first its attachments to the growth substratum. These findings are consistent with the 12(S)-HETE-induced loss of paxillin from adhesion sites that we observed in pseudopods of carcinoma cells (Ross et al., 2000).

Structurally, the integrity of adhesion sites and that of the F-actin cytoskeleton are known to be interdependent (Critchley et al., 1999; Small et al., 1999). Our data suggest, however, that growth cone collapse depends to a significant degree on the disassembly and detachment of adhesion sites, via a signaling pathway that may be partially distinct from that regulating the actin cytoskeleton. Support for this interpretation stems from the preferential inhibition of adhesion site disassembly by CDC, as shown here, and from the fact that thrombin-induced growth cone detachment does not require a functional actin cytoskeleton (de la Houssaye et al., 1999). Our observations suggest, furthermore, that the $12 / 15-\mathrm{LO}$ product, $12(\mathrm{~S})$-HETE, affects primarily the adhesion sites.

The search for molecules that mediate Sema-induced growth cone collapse has identified a number of potential signaling elements. Members of the Rho family of GTPases, especially Rac1, have been implicated in collapse (Jin and Strittmatter, 1997; Zanata et al., 2002). Rac1 seems to control the changes of the actin cytoskeleton associated with collapse (Kuhn et al., 1999). However, Rho family GTPases also are necessary for growth cone spreading (Mackay et al., 1995), and Rho may be more important for neurite retraction than collapse (Arimura et al., 2000). Also, inhibition of Rho blocks lysophosphatidic acid-mediated growth cone collapse in neuronal cell lines (Jalink et al., 1994) but causes growth cone collapse in sensory neurons (Jin and Strittmatter, 1997). There is no doubt that Rho family GTPases are involved in the cytoskeletal reorganizations accompanying growth cone spreading and collapse, but it remains unclear whether they are steps in the repellent receptor-activated signaling cascade. The CRMPs (Goshima et al., 1995) have been implicated in collapse but today seem more likely to regulate microtubule dynamics and axon formation (Gu and Ihara, 2000; Inagaki et al., 2001). Therefore, a consistent picture of repellent signaling has failed to emerge so far.

How 12(S)-HETE regulates the disassembly of adhesion sites and collapse is unknown. Is there cross talk between 12(S)HETE generation and the rearrangement of the actin cytoskeleton? Interestingly, 12(S)-HETE has been implicated indirectly in Rac1 signaling (Wen et al., 2000). This could explain our observation that $12(\mathrm{~S})$-HETE treatment of cultured DRG growth cones causes partial F-actin loss, in addition to adhesion site disassembly. However, our own preliminary data suggest that 12(S)-HETE activates a PKC isoform, which in turn leads to the phosphorylation of the adhesion site protein myristoylated alanine-rich C-kinase substrate (our unpublished observations), a protein involved in cell spreading (Rosen et al., 1990; Myat et al., 1997; Laux et al., 2000).

\section{Conclusions}

Our data demonstrate that Sema3A treatment of DRG growth cones stimulates the synthesis of 12(S)-HETE and that its generation is necessary for growth cone collapse and detachment. 12(S)-HETE mimics, and therefore is sufficient to cause, collapse when added to DRG growth cones exogenously. Furthermore, our evidence suggests that increases in 12(S)-HETE levels cause a reduction of growth cone adhesion. These observations are consistent with our previous finding that thrombin-treated corti- cal neurons require 12/15-LO activity for collapse and that collapse involves regulated detachment. Therefore, the generation of 12(S)-HETE and the regulation of adhesion sites may be general phenomena of repellent signaling.

\section{REFERENCES}

Arimura N, Inagaki N, Chihara K, Menager C, Nakamura N, Amano M, Iwamatsu A, Goshima Y, Kaibuchi K (2000) Phosphorylation of collapsin response mediator protein-2 by Rho-kinase. Evidence for two separate signaling pathways for growth cone collapse. J Biol Chem 275:23973-23980.

Behar O, Mizuno K, Badminton M, Woolf CJ (1999) Semaphorin 3A growth cone collapse requires a sequence homologous to tarantula hanatoxin. Proc Natl Acad Sci USA 96:13501-13505.

Cho H, Ueda M, Tamaoka M, Hamaguchi M, Aisaka K, Kiso Y, Inoue T, Ogino R, Tatsuoka T, Ishihara T, Noguchi T, Morita I, Murota S (1991) Novel caffeic acid derivatives: extremely potent inhibitors of 12-lipoxygenase. J Med Chem 34:1503-1505.

Critchley DR, Holt MR, Barry ST, Priddle H, Hemmings L, Norman J (1999) Integrin-mediated cell adhesion: the cytoskeletal connection. Biochem Soc Symp 65:79-99.

Dailey LA, Imming P (1999) 12-Lipoxygenase: classification, possible therapeutic benefits from inhibition, and inhibitors. Curr Med Chem 6:389-398.

de la Houssaye BA, Mikule K, Nikolic D, Pfenninger KH (1999) Thrombin-induced growth cone collapse: involvement of phospholipase $A_{2}$ and eicosanoid generation. J Neurosci 19:10843-10855.

Doukas J, Hechtman HB, Shepro D (1988) Endothelial-secreted arachidonic acid metabolites modulate polymorphonuclear leukocyte chemotaxis and diapedesis in vitro. Blood 71:771-779.

Fan J, Raper JA (1995) Localized collapsing cues can steer growth cones without inducing their full collapse. Neuron 14:263-274.

Fournier AE, Kalb RG, Strittmatter SM (2000a) Rho GTPases and axonal growth cone collapse. Methods Enzymol 325:473-482.

Fournier AE, Nakamura F, Kawamoto S, Goshima Y, Kalb RG, Strittmatter SM (2000b) Semaphorin3A enhances endocytosis at sites of receptor-F-actin colocalization during growth cone collapse. J Cell Biol 149:411-422.

Fritsche J, Reber BF, Schindelholz B, Bandtlow CE (1999) Differential cytoskeletal changes during growth cone collapse in response to hSema III and thrombin. Mol Cell Neurosci 14:398-418.

Goshima Y, Nakamura F, Strittmatter P, Strittmatter SM (1995) Collapsin-induced growth cone collapse mediated by an intracellular protein related to UNC-33. Nature 376:509-514.

Goshima Y, Hori H, Sasaki Y, Yang T, Kagoshima-Maezono M LiC, Takenaka T, Nakamura F, Takahashi T, Strittmatter SM, Misu Y, Kawakami T (1999) Growth cone neuropilin-1 mediates collapsin-1) Sema III facilitation of antero- and retrograde axoplasmic transport. J Neurobiol 39:579-589.

$\mathrm{Gu}$ Y, Ihara Y (2000) Evidence that collapsin response mediator protein-2 is involved in the dynamics of microtubules. J Biol Chem 275:17917-17920.

Inagaki N, Chihara K, Arimura N, Menager C, Kawano Y, Matsuo N, Nishimura T, Amano M, Kaibuchi K (2001) CRMP-2 induces axons in cultured hippocampal neurons. Nat Neurosci 4:781-782.

Izzard CS, Lochner LR (1976) Cell-to-substrate contacts in living fibroblasts: an interference reflexion study with an evaluation of the technique. J Cell Sci 21:129-159.

Jalink K, Eichholtz T, Postma FR, van Corven EJ, Moolenaar WH (1993) Lysophosphatidic acid induces neuronal shape changes via a novel, receptor-mediated signaling pathway: similarity to thrombin action. Cell Growth Differ 4:247-255.

Jalink K, van Corven EJ, Hengeveld T, Morii N, Narumiya S, Moolenaar WH (1994) Inhibition of lysophosphatidate- and thrombin-induced neurite retraction and neuronal cell rounding by ADP ribosylation of the small GTP-binding protein Rho. J Cell Biol 126:801-810.

Jin Z, Strittmatter SM (1997) Rac1 mediates collapsin-1-induced growth cone collapse. J Neurosci 17:6256-6263.

Keynes RJ, Cook GM (1992) Repellent cues in axon guidance. Curr Opin Neurobiol 2:55-59.

Kuhn TB, Brown MD, Wilcox CL, Raper JA, Bamburg JR (1999) Myelin and collapsin-1 induce motor neuron growth cone collapse through different pathways: inhibition of collapse by opposing mutants of rac1. J Neurosci 19:1965-1975.

Lauffenburger DA, Horwitz AF (1996) Cell migration: a physically integrated molecular process. Cell 84:359-369.

Laux T, Fukami K, Thelen M, Golub T, Frey D, Caroni P (2000) GAP43, MARCKS, and CAP23 modulate PI(4, 5)P(2) at plasmalemmal rafts, and regulate cell cortex actin dynamics through a common mechanism. J Cell Biol 149:1455-1472.

Mackay DJ, Nobes CD, Hall A (1995) The Rho's progress: a potential role during neuritogenesis for the Rho family of GTPases. Trends Neurosci 18:496-501. 
Mazurov AV, Leytin VL, Repin VS, Smirnov VN, Forster W (1983) Arachidonic acid and stable analogue of prostaglandin endoperoxides (U46619) induce platelet spreading and thrombin-like aggregate formation on a collagen substrate. Effect of fluid dynamics. Thromb Res 32:189-205.

Myat MM, Anderson S, Allen LA, Aderem A (1997) MARCKS regulates membrane ruffling and cell spreading. Curr Biol 7:611-614.

Negre-Aminou P, Pfenninger KH (1993) Arachidonic acid turnover and phospholipase A2 activity in neuronal growth cones. J Neurochem 60:1126-1136.

Pfenninger KH, Maylie-Pfenninger MF (1981) Lectin labeling of sprouting neurons. II. Relative movement and appearance of glycoconjugates during plasmalemmal expansion J Cell Biol 89:547-559.

Reza JN, Gavazzi I, Cohen J (1999) Neuropilin-1 is expressed on adult mammalian dorsal root ganglion neurons and mediates semaphorin $3 \mathrm{a} /$ collapsin-1-induced growth cone collapse by small diameter sensory afferents. Mol Cell Neurosci 14:317-326.

Richards LJ, Koester SE, Tuttle R, O'Leary DD (1997) Directed growth of early cortical axons is influenced by a chemoattractant released from an intermediate target. J Neurosci 17:2445-2458.

Rosen A, Keenan KF, Thelen M, Nairn AC, Aderem A (1990) Activation of protein kinase $\mathrm{C}$ results in the displacement of its myristoylated, alanine-rich substrate from punctate structures in macrophage filopodia. J Exp Med 172:1211-1215.

Ross S, Essary B, de la Houssaye BA, Pan Z, Mikule K, Mubarak O, Pfenninger KH (2000) Thrombin causes pseudopod detachment via a pathway involving cytosolic phospholipase A2 and 12/15-lipoxygenase products. Cell Growth Differ 11:19-30.
Small JV, Rottner K, Kaverina I (1999) Functional design in the actin cytoskeleton. Curr Opin Cell Biol 11:54-60.

Soker S, Takashima S, Miao HQ, Neufeld G, Klagsbrun M (1998) Neuropilin-1 is expressed by endothelial and tumor cells as an isoformspecific receptor for vascular endothelial growth factor. Cell 92:735-745.

Song H, Ming G HeZ, Lehmann M, McKerracher L, Tessier-Lavigne M, Poo M (1998) Conversion of neuronal growth cone responses from repulsion to attraction by cyclic nucleotides. Science 281:1515-1518.

Takahashi T, Fournier A, Nakamura F, Wang LH, Murakami Y, Kalb RG, Fujisawa H, Strittmatter SM (1999) Plexin-neuropilin-1 complexes form functional semaphorin-3A receptors. Cell 99:59-69.

Taniguchi M, Yuasa S, Fujisawa H, Naruse I, Saga S, Mishina M, Yagi T (1997) Disruption of semaphorin III/D gene causes severe abnormality in peripheral nerve projection. Neuron 19:519-530.

Tessier-Lavigne M, Goodman CS (1996) The molecular biology of axon guidance. Science 274:1123-1133.

Wen Y, Gu J, Knaus UG, Thomas L, Gonzales N, Nadler JL (2000) Evidence that 12-lipoxygenase product 12-hydroxyeicosatetraenoic acid activates p21-activated kinase. Biochem J 349:481-487.

Yamamoto S, Suzuki H, Nakamura M, Ishimura K (1999) Arachidonate 12-lipoxygenase isozymes. Adv Exp Med Biol 447:37-44.

Zanata SM, Hovatta I, Rohm B, Puschel AW (2002) Antagonistic effects of Rnd1 and RhoD GTPases regulate receptor activity in Semaphorin 3A-induced cytoskeletal collapse. J Neurosci 15:471-477.

Zhou FQ, Cohan CS (2001) Growth cone collapse through coincident loss of actin bundles and leading edge actin without actin depolymerization. J Cell Biol 153:1071-1084. 